\title{
THE EXISTENCE FOR THE SOLUTION OF THE ELLIPTIC CAUCHY PROBLEM
}

\author{
BY CHUNG-LING YU
}

Communicated by François Treves, October 24, 1973

Let $D$ be a simply connected domain of the $z=x+i y$ plane, whose boundary contains a portion $\sigma$ of the $x$-axis. Also let $A(z, \zeta), B(z, \zeta)$ be holomorphic functions for $z, \zeta \in D \cup \sigma \cup \bar{D}$, where $\bar{D}=\{z \mid \bar{z} \in D\}$. The aim of this note is to announce some recent results on the global existence for the Cauchy problem of the first order linear elliptic equations (in complex normal form):

$$
\partial W / \partial \bar{z}=A(z, \bar{z}) W+B(z, \bar{z}) \bar{W}
$$

where

$$
\partial / \partial \bar{z}=\frac{1}{2}(\partial / \partial x+i \partial / \partial y) \cdot W=u+i v .
$$

The notations in this note are taken from $\mathrm{Yu}$ [8], [9]. For general reference to the elliptic Cauchy problem, the reader is referred to the survey article by Payne [6], and to equation (1), the reader is referred to Vekua [7] and $\mathrm{Yu}[8]$, [9].

By using transformation

$$
W(z, \bar{z})=W_{0}(z, \bar{z}) \exp \int_{\zeta_{1}}^{\bar{z}} A(z, t) d t
$$

where $\zeta_{1}$ is a fixed point in $\bar{D}$, the equation (1) can be reduced to the form

(2) $\frac{\partial W_{0}}{\partial \bar{z}}=c(z, \bar{z}) \bar{W}_{0} ; c(z, \bar{z})=B(z, \bar{z}) \exp \left[\int_{\xi_{1}}^{z} A^{*}(\bar{z}, t) d t-\int_{\zeta_{1}}^{z} A(z, t) d t\right]$.

The following integral representation for the solution of (1) in a simply connected domain $G \subset D \cup \sigma \cup \bar{D}$ has been established by Vekua [7], and later extended to the boundary $\partial G$ of $G$ by $\mathrm{Yu}$ [9].

LEMMA 1. Every solution $W(z)$ of (1) in $G$, continuous in $G \cup \partial G$, has the integral representation

$$
\begin{aligned}
W(z)=\{\phi(z) & +\int_{z 0}^{z} \Gamma_{1}\left(z, \bar{z}, t, \zeta_{0}\right) \phi(t) d t \\
& \left.+\int_{\zeta_{0}}^{z} \Gamma_{2}\left(z, \bar{z}, z_{0}, \tau\right) \phi^{*}(\tau) d \tau\right\} \exp \int_{\zeta_{1}}^{z} A(z, t) d t,
\end{aligned}
$$

AMS (MOS) subject classifications (1970). Primary 30A92, 30A93, 30A82, 35A20, 35 B30, 35G10, 35J60.

Key words and phrases. Cauchy problem, elliptic partial differential equations. 
where $\phi(z)$ is a function holomorphic in $G$, and continuous in $G \cup \partial G$. $\Gamma_{1}(z, \zeta, t, \tau), \Gamma_{2}(z, \zeta, t, \tau)$ are holomorphic for $z, \zeta, t, \tau \in D \cup \sigma \cup \bar{D}$, and can be constructed explicitly by Vekua [7]. Conversely, if $W(z)$ is given by (3), then $W(z)$ is a solution of (1) in $G$, and continuous in $G \cup \partial G$.

We shall also make use of the following generalized Cauchy integral formula for (2).

Lemma 2. Let $G$ be a simply connected domain with piecewise smooth boundary $\partial G$ such that $G \cup \partial G \subset D \cup \sigma \cup \bar{D}$, then for any solution $W_{0}(z)$ of (2) in $G$, continuous in $G \cup \partial G$, we have

$$
W_{0}(z)=\frac{1}{2 \pi i} \int_{\partial G}[W(t) U(t, \bar{t}, z, \bar{z}) d t-(\bar{W}(t))(\bar{V}(t, \bar{t}, z, \bar{z})) d \bar{t}]
$$

where

$$
\begin{aligned}
U & =U_{1}-U_{2}, \quad V=V_{1}-V_{2}, \\
U_{1} & =1 /(t-z)-\Gamma_{1}(z, \bar{z}, t, \bar{t}) \log [(t-z)(\bar{t}-\bar{z})]+\Sigma_{1}, \\
V_{1} & =-\Gamma_{2}^{*}(\bar{z}, z, t, \bar{t}) \log [(t-z)(\bar{t}-\bar{z})]+\Sigma_{2},
\end{aligned}
$$

and $\Sigma_{1}(t, \eta, z, \zeta), \Sigma_{2}(t, \eta, z, \zeta), U_{2}(z, \eta, z, \zeta), V_{2}(t, \eta, z, \zeta)$ are certain holomorphic functions of $t, \eta, z, \zeta \in D \cup \sigma \cup \bar{D}$, given explicitly in Vekua [7].

Since the principal part of (1) is invariant under conformal mapping, it is no loss of generality to state the Cauchy problem for equation (1) as the following: To find a solution $w(z)$ of (1) satisfying the following condition

$$
w(x)=\rho(x), \quad x \in \sigma,
$$

where $\rho(x)$ is continuous on $\sigma$.

The following theorem gives a method of numerical integration for an analytic data $\rho(x)$.

THEOREM 1. Let the analytic continuation $\rho(z)$ of $\rho(x)$ be holomorphic throughout $D \cup \sigma \cup \bar{D}$. Then the solution of the Cauchy problem for (1) is analytic for $(x, y)$ in $D \cup \sigma \cup \bar{D}$, and can be constructed by the method of successive approximation.

SKETCH OF THE PROOF. Solve the equation

$$
\begin{aligned}
\rho(z)=\{\phi(z) & +\int_{0}^{z} \Gamma_{1}(z, z, t, 0) \phi(t) d t \\
& \left.+\int_{0}^{z} \Gamma_{2}(z, z, 0, \tau) \phi^{*}(\tau) d \tau\right\} \exp \int_{\zeta_{1}}^{z} A(z, t) d t
\end{aligned}
$$


by the method of successive approximation for an unknown function $\phi(z)$. Hence the desired result follows from (3).

REMARK 1. The above method gives the integral representation for the solution in a symmetric domain (or conformal symmetric domain), and information of continuous dependence. This theorem can be considered as a continuation of Hadamard [4], Garabedian [3], Henrici [5], Colton [2], and Aziz, Gilbert, Howard [1].

Theorem 2 below establishes a necessary and sufficient condition for the existence of a solution of problems (2) and (5), and also gives a method for constructing the global solution. We shall make use of the following lemma.

LEMMA 3. Let

$$
G(z)=\frac{1}{2 \pi i} \int_{\sigma} \rho(t) U d t-(\bar{\rho}(t)) \bar{V} d t .
$$

Then $G(z)$ is a solution of (2) in $D \cup \bar{D}$ (off $\sigma)$. Furthermore, if $\rho(\tau)$ is Hölder continuous on $\sigma$, then

$$
G^{+}(x)=\frac{1}{2} \rho(x)+G(x), \quad x \in \sigma
$$

where $G^{+}(x)$ is the limit function of $G(z)$ for $z \in D$ (we assume $D$ is on the left of $\sigma)$, and $G(x)$ being understood as the Cauchy principal value.

Proof. By direct calculation (cf. Vekua [7, p. 81]).

THEOREM 2. Let

$$
F(x)=\rho(x)-G^{+}(x) .
$$

Then $\rho(x)=W_{0}(x)$ is a Cauchy data of a solution $W_{0}(z)$ of $(2)$ in $D$ if and only if $F(x)$ is analytic and its analytic continuation $F(z)$ is a holomorphic function in $D \cup \sigma \cup \bar{D}$.

SKETCH OF THE PROOF. Let

$$
H(z, \bar{z})=W_{0}(z)-G(z)=\frac{1}{2 \pi i} \int_{\partial D \backslash \sigma} W_{0} U d t-\bar{W}_{0} \bar{V} d \bar{t} .
$$

Then $W_{0}(z)$ is a solution in $D$ if and only if $H(z, z)=F(z)$ is holomorphic in $D \cup \sigma \cup \bar{D}$.

REMARK 2. By Theorem 1. We can construct a solution $H(z, \bar{z})$ of (2) in $D \cup \sigma \cup \bar{D}$ such that $H(x, x)=F(x)$. Hence $W_{0}(z)=H(z, \bar{z})+G(z)$ is the solution of the Cauchy problem (2) and (5) in $D$.

REMARK 3. Theorem 2 is a generalization of Hadamard [4].

REMARK 4. Since the principal part of (1) is invariant under conformal mapping, the above theorem can be extended to a more general domain with nonanalytic initial curve. 
REMARK 5. In a forthcoming paper [10], we shall give another version of necessary and sufficient conditions for existence. We shall also study the estimate of the 'stability' and the construction for an approximate solution with imprecise data.

REMARK 6. The results in this note can be extended to equation (1) with nonanalytic coefficients [12] and to some higher order elliptic equations [11].

\section{REFERENCES}

1. A. K. Aziz, R. P. Gilbert and H. C. Howard, A second order, non-linear elliptic boundary value problem with generalized Goursat data, Ann. Mat. Pura Appl. (4) 72 (1966), 325-341. MR 34 \#4697.

2. D. Colton, Cauchy's problem for almost linear elliptic equations in two independent variables, J. Approximation Theory 3 (1970), 66-71. MR 41 \#3985.

3. P. R. Garabedian, Partial differential equations, Wiley, New York, 1964. MR 28 \#5247.

4. J. Hadamard, Lectures on the Cauchy problem in linear partial differential equations, Yale Univ. Press, New Haven, Conn., 1923.

5. P. Henrici, A survey of $I$. $N$. Vekua's theory of elliptic partial differential equations with analytic coefficients, Z. Angew. Math. Phys. 8 (1957), 169-203. MR 19, 38.

6. L. E. Payne, Some remarks on improperly posed problems for partial differential equations, Lecture Notes in Math., vol. 316, Springer-Verlag, Berlin and New York, 1973, pp. 1-30.

7. I. N. Vekua, New methods for solving elliptic equations, OGIZ, Moscow, 1948; English transl., Series in Appl. Math., vol. 1, North-Holland, Amsterdam; Interscience, New York, 1967. MR 11, 598; 35 \#3243.

8. C. L. Yu, Reflection principle for systems of first order elliptic equations with analytic coefficients, Trans. Amer. Math. Soc. 164 (1972), 489-501. MR 45 \#2189.

9. - Cauchy problem and analytic continuation for systems of first order elliptic equations with analytic coefficients, Trans. Amer. Math. Soc. 185 (1973), 429-443.

10. - Cauchy problem for systems of first order analytic elliptic equations, SIAM J. Math. Anal. (to appear).

11. - Cauchy problem for a class of higher order analytic elliptic equations (to appear).

12. - On the global solvability of a linear elliptic Cauchy problem (to appear).

Department of Mathematics, Florida State University, Tallahassee, Florida 32306 